\title{
FORMULATION OF POLYACRYLIC ACID-LAYERED DOUBLE HYDROXIDE COMPOSITE SYSTEM AS A SOIL CONDITIONER FOR WATER MANAGEMENT
}

\author{
INAS H. HAFEZ \\ Institute of Technology and Science, The University of Tokushima, \\ Tokushima 770-8506, Japan. \\ Ihazzaa@sy34.chem.tokushima-u.ac.jp \\ MOHAMED R. BERBER \\ Department of Chemistry, Faculty of Science, Tanta University, \\ Tanta 31527, Egypt. \\ mrberber@science.tanta.edu.eg \\ KEIJI MINAGAWA \\ Institute of Technology and Science, The University of Tokushima, \\ Tokushima 770-8506, Japan. \\ minagawa@chem.tokushima-u.ac.jp \\ TAKESHI MORI \\ Department of Applied Chemistry, Kyushu University, \\ Fukuoka 819-0395, Japan. \\ mori.takeshi.880@m.kyushu-u.ac.jp \\ MASAMI TANAKA \\ Faculty of Pharmaceutical Sciences, Tokushima Bunri University, \\ Tokushima 770-8514, Japan. \\ tanaka@ph.bunri-u.ac.jp
}

\begin{abstract}
An efficient strategy of soil-conditioner application was offered by incorporating a high molecular weight organic polymer (polyacrylic acid; PAA) into a soil-friendly inorganic material (layered double hydroxide; LDH). The prepared materials were characterized by different spectroscopic techniques to confirm the formed nanocomposite structure. The SEM images captured the morphological ability of PAA-LDH nanocomposites to absorb and keep water molecules during soil-water application. The IR analysis indicated an electrostatic grafting process between PAA units and LDH moieties. The platform of PAA-LDH nanocomposite formulation stabilized the soil aggregates and improved the water-stability.
\end{abstract}

Keywords: Soil conditioners; nanocomposite; LDH. 


\section{Introduction}

Water management strategies are closely related to management of water resources. A recent strategy of water management is the use of high molecular weight polymers to soil as a mean to retain the structure of the soil and to decrease the water infiltration ${ }^{1}$. The decrease of water infiltration has several positive effects such as maintaining soil moisture and soil productivity. However the application of synthetic organic polymers as soil conditioners may affect the quality of groundwater, planting process and even soil itself. Thus, there is a need in-the-art of new technology to save the application of organic polymers in soils.

In recent years, absorptive polymer/clay composites have received a great attention because of their relative low production costs and high water absorbitivity ${ }^{2}$. Polyacrylate polymers generally exhibit a very high absorbency in water, however they have the problem of poor resistance to salts, that decreases its soil-application ${ }^{3}$. Layered double hydroxide (LDHs) are a family of natural and synthetic compounds, consists structurally of brucite-like sheets with hexagonal-shaped crystallite layers ${ }^{4}$. It possesses excellent anion exchanging properties and can be used as matrixes, polymer stabilizers and carriers. In addition, $\mathrm{LDH}$ is reported in a number of studies as a soil biocompatible and nontoxic material $^{5,6}$.

Herein we offer an efficient strategy of polymer-soil application by incorporating an anionic high molecular weight polymer (polyacrylic acid, PAA) into a soil-friendly inorganic material; LDH. The formation of organic-inorganic composites of PAA with LDH is expected to improve the soils-application of PAA.

\section{Experimental Work}

\subsection{Materials}

Acrylic acid monomer (distilled under reduced pressure prior to use), ammonium persulphate (analytical grade), magnesium nitrate hexahydrate (purity 99.5\%), aluminum nitrate nanohydrate (purity 99.5\%) were purchased from Wako Pure Chemical Industries, Ltd. and used as received. All solutions were prepared using deionized water (18.2 $\mathrm{M} \Omega / \mathrm{cm}$, produced from Milli-Q Grandient ZMQG000kt).

\subsection{Preparation of $\mathrm{NO}_{3}-\mathrm{LDH}$}

To a round flask containing $30 \mathrm{~mL}$ of $1.0 \mathrm{M} \mathrm{NaNO}_{3}$ solution was added dropwise a 50 $\mathrm{mL}$ solution of $8.538 \mathrm{~g}(0.0333 \mathrm{M})$ of $\mathrm{Mg}\left(\mathrm{NO}_{3}\right)_{2} \cdot 6 \mathrm{H}_{2} \mathrm{O}$ and $6.249 \mathrm{~g}(0.01666 \mathrm{M})$ of $\mathrm{Al}\left(\mathrm{NO}_{3}\right)_{3} \cdot 9 \mathrm{H}_{2} \mathrm{O}\left(\mathrm{Mg}^{2+} / \mathrm{Al}^{3+}=2\right)$ with constant stirring. During the metal ion addition, the $\mathrm{pH}$ of the suspension was kept constant at 8.0 by adding appropriate amounts of $2.0 \mathrm{M}$ $\mathrm{KOH}$ solution. The final volume of the preparation medium was augmented to $100 \mathrm{~mL}$ by deionized water. The resultant suspension was stirred at $70{ }^{\circ} \mathrm{C}$ for $24 \mathrm{~h}$ under $\mathrm{N}_{2}$ flow. At the end of the reaction, the formed $\mathrm{Mg}-\mathrm{Al} \mathrm{LDH}$ material was collected by filtration, washed several times with deionized water, and finally freeze-dried. 


\subsection{Nanohybrid synthesis of AA-LDH by anion-exchange technique.}

In a $100 \mathrm{~mL}$ round flask was dissolved $2.0 \mathrm{~g}$ of AA in $100 \mathrm{~mL}$ of deionized water by adjusting the solution $\mathrm{pH}$ to 8.0 using $2.0 \mathrm{M} \mathrm{NaOH}$ solution. To the AA solution, $1.0 \mathrm{~g}$ of $\mathrm{NO}_{3}$ - $\mathrm{LDH}$ was added with constant stirring. The reaction mixture was stirred at $40{ }^{\circ} \mathrm{C}$ for 7 days. After the reaction, the obtained AA-LDH material was collected by filtration (0.45 $\mu \mathrm{m}$ Millipore membrane) and washed several times by $0.1 \mathrm{M} \mathrm{NaOH}$ solution and subsequently deionized water. Finally, the collected AA-LDH was freeze-dried.

\subsection{In-situ polymerizations of AA-LDH nanocomposites}

A flask containing $5.0 \mathrm{~g}$ AA-LDH and a $0.5 \mathrm{wt} \%$ of ammonium persulphate initiator in a $100 \mathrm{~mL}$ deionized water was degassed under $\mathrm{N}_{2}$ vacuum by three freeze-thaw cycles. The reaction mixture was stirred and heated to $80{ }^{\circ} \mathrm{C}$ in a water bath for $3 \mathrm{~h}(\mathrm{~s})$. After the polymerization reaction, the PAA-LDH material was collected by filtration, washed several times by $0.1 \mathrm{M} \mathrm{NaOH}$ solution and subsequently deionized water. Finally, the collected PAA-LDH was freeze-dried.

\subsection{Characterization and measurements}

$\mathrm{X}$-ray powder diffraction patterns were recorded on a Rigaku X-ray diffractometer using $\mathrm{CuK} \alpha$ radiation at $\lambda=1.5405 \AA$. The measurement was performed in the $2 \theta$ range $1.5-70^{\circ}$. Infrared spectra (KBr disk method) were recorded on a Bio-Rad FTS 3000MX FT-IR spectrophotometer. The scanning electron micrographs (SEM) were captured by a Hitachi FE-SEMS-4700 microscope.

Soil samples with a mass of $100 \mathrm{~g}$ were placed in battery dishes. PAA and PAA-LDH emulsions with $0.2 \mathrm{wt} \%$ were sprayed on the surface of the soil. The sprayed soils were remained two days to dry at room temperature. The static water-measure technique was used to determine the water-stability of the soil aggregates. Admixed aggregates of similar diameter (visually approximated) were then selected and replaced on a filter paper in new battery dishes. The soil aggregates were immersed in water. The time of immersion until collapse was recorded. The collapse is considered when interior water began to effuse. The average values of triplicate measurements were obtained. The water-stability test was repeated by using 0.4 and $0.6 \mathrm{wt} \%$ of PAA and PAA-LDH.

\section{Results and discussion}

The scanning electron microscopy was used to investigate the nanocomposite structure of the prepared materials. Figure 1 displays the SEM images of the synthesized $\mathrm{NO}_{3}-\mathrm{LDH}$ material, AA-LDH and PAA-LDH composites. The inset of image a, shows uniform and regular hexagonal platelets characteristic for $\mathrm{LDH}$ particles. The regularity of the LDH particles confirmed the good crystallinity recorded by X-ray measurement. In the case of AA-LDH (images b), aggregates composed of small nanoparticles were observed. On the other side, PAA-LDH (images c), showed a considerable difference. The surface of the 
aggregates exhibited a porous morphological structure. Hence, the PAA-LDH material can swell through the diffusion of the water molecules into the pores of the nanocomposites structure. In conclusion, the recorded SEM images confirmed the nanocomposite structure of PAA-LDH and captured the morphological ability of PAA-LDH to absorb and keep water molecules during soil-water application.
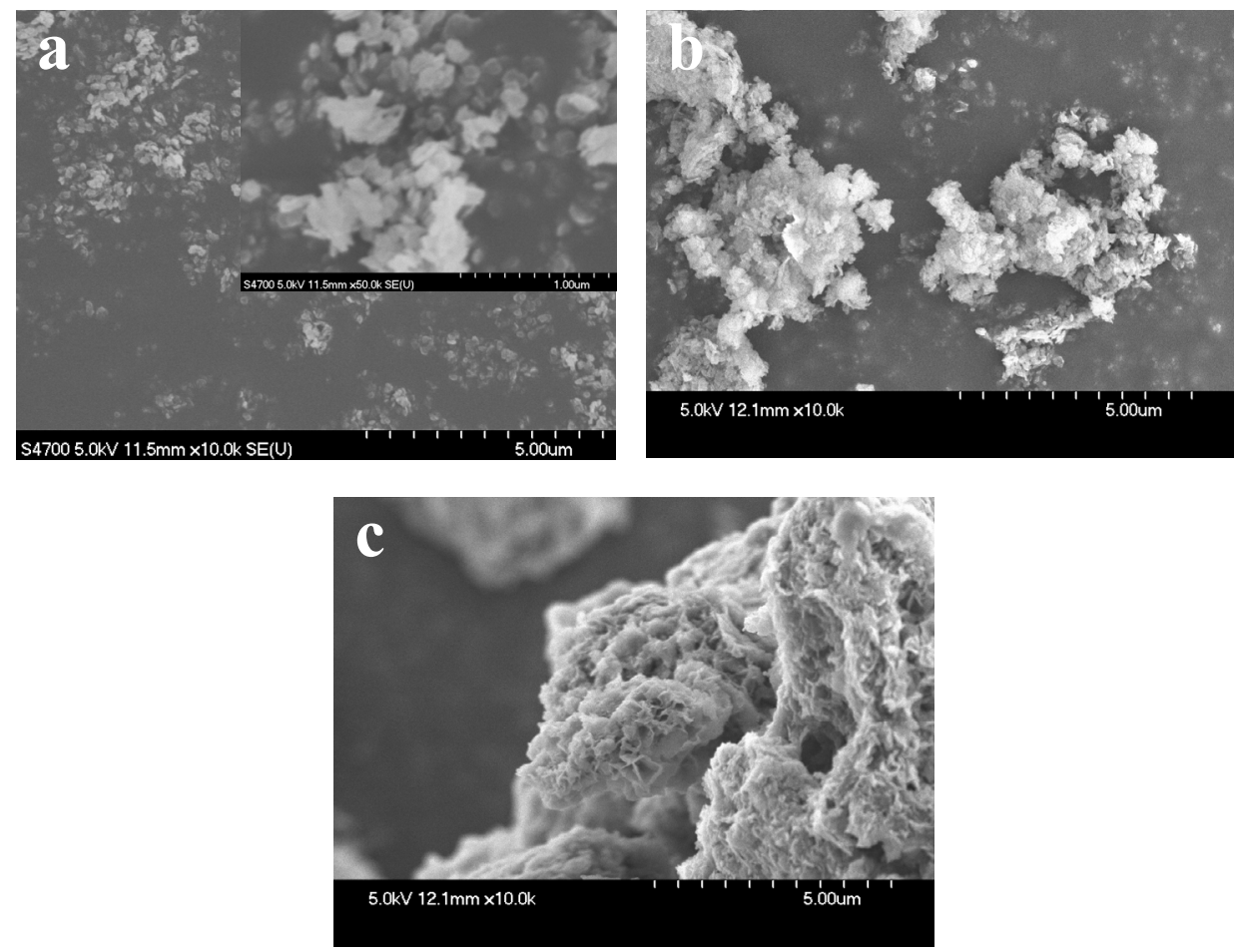

Fig. 1. SEM images of $\mathrm{NO}_{3}$-LDH (a), AA-LDH (b), and PAA-LDH (c) hybrids

Figure 2 shows the XRD patterns of LDH, AA-LDH and PAA-LDH nanocomposites. Pattern $\mathrm{A}$ indicates the formation of the $\mathrm{NO}_{3}$ form of $\mathrm{LDH}$ with sharp and symmetric (00l) reflections. Using Bragg's law ( $\mathrm{n} \lambda=2 \mathrm{~d} \sin \theta$ ), the basal $\mathrm{d}$-spacing was calculated to be $8.9 \AA$. The anion exchange process of AA anions resulted in a shift to a lower angle with an increase in the basal d-spacing. The calculated gallery height was found to be $12.6 \AA$ (pattern B). The AA-LDH nanocomposites interlayer distance was calculated by subtracting the inorganic layer thickness $(4.8 \AA)^{7}$ from the $\mathrm{d}_{003}$-spacing. The determined value $(7.8 \AA)$ was slightly larger than AA molecular length $(6.0 \AA)$. However, LDH attaches its interlayer anions through an electrostatic interaction. Thus, we speculated that AA molecules were stacked into $\mathrm{LDH}$ as a monolayer through the attachment of AA carboxyl groups with the LDH positive layers. In the case of PAA-LDH (pattern c), the 
basal spacing reflections are broadened, indicating a turbostratic disorder of the LDH layers. This result was an indication of the stacking polymerization process of AA-LDH.

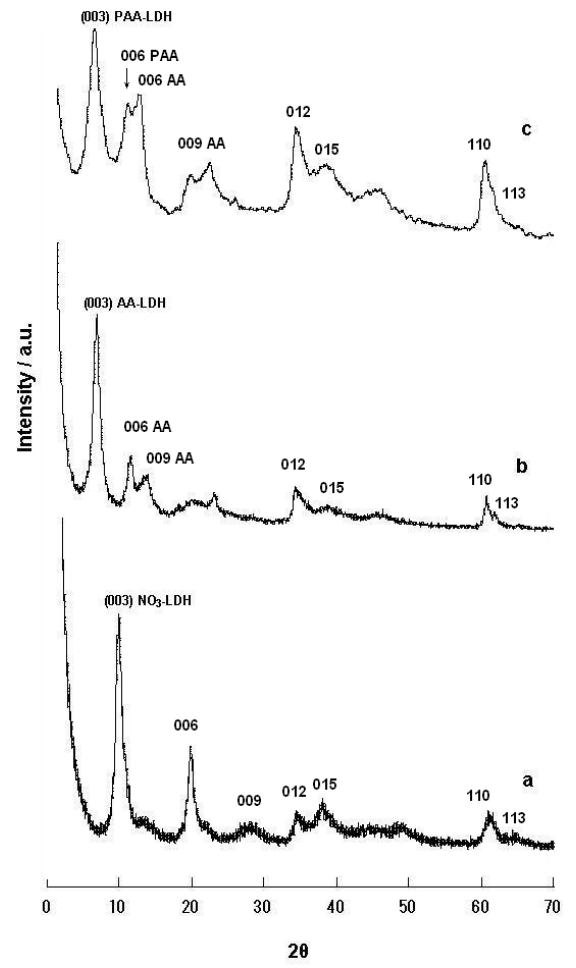

Fig. 2. X-ray diffraction patterns of $\mathrm{NO}_{3}$-LDH (a), AA-LDH (b), PAA-LDH (c)

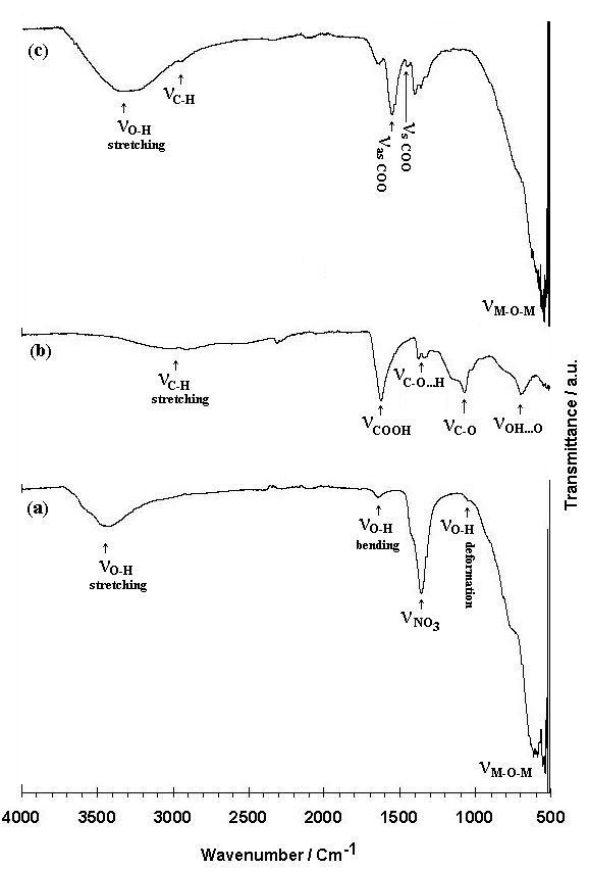

Figure 3. Infrared spectra of $\mathrm{NO}_{3}-\mathrm{LDH}(\mathrm{a})$,

PAA (b), and PAA-LDH (c)

Figure 3a exhibited the characteristic bands of $\mathrm{NO}_{3}-\mathrm{LDH}$, in particular the lattice vibration modes ${ }^{8}\left(v_{\mathrm{M}-\mathrm{O}-\mathrm{M}}, v_{\mathrm{M}-\mathrm{O}}\right)$ at 540,630 , and $775 \mathrm{~cm}^{-1}$ and the asymmetric stretching band of the interlayer nitrate $\left(v_{\mathrm{NO} 3}\right)$ at $1365 \mathrm{~cm}^{-1}$ as well as the vibration modes of the hydroxyl groups of LDH layers ; bending at $1050 \mathrm{~cm}^{-1}$, deformation at $1660 \mathrm{~cm}^{-1}$ and stretching at $3450 \mathrm{~cm}^{-1}$. Figure $3 \mathrm{~b}$ showed the characteristic features of $\mathrm{PAA}^{10}$. The stretching vibration modes of alkyl groups $\left(v_{\mathrm{C}-\mathrm{H}}\right)$ were detected at $3030 \mathrm{~cm}^{-1}$. The stretching vibration band of $\mathrm{COOH}$ group was assigned at $1709 \mathrm{~cm}^{-1}$. The combinations of the in-plane deformation modes of $\mathrm{C}-\mathrm{O} \ldots \mathrm{H}$ and the stretching vibration modes of $\mathrm{C}-\mathrm{O}$ were recorded at $1400-1350 \mathrm{~cm}^{-1}$ and $1150-1100 \mathrm{~cm}^{-1}$, respectively. The broad band at $800 \mathrm{~cm}^{-1}$ is associated to an out-of-plane $\mathrm{OH} . . \mathrm{O}$ deformation, indicating a strong intermolecular hydrogen bonding. As a result of intercalation of PAA with LDH (spectrum c), new bands are emerged such as asymmetric and symmetric modes of $\mathrm{COO}^{-}$ at 1560 and $1460 \mathrm{~cm}^{-1}$, respectively, besides the characteristic bands of PAA and LDH. 
The lack of the inorganic anion $\left(\mathrm{NO}_{3}\right)$ in the interlayer gallery indicated the formation of monophase nanocomposites of PAA-LDH.

The FT-IR data emphasized the grafting between PAA and LDH through electrostatic interaction. Such kind of interaction enables LDH nanoparticles to act as network points in the composite structure after the polymerization process of AA units. In addition, this crosslinking behaviour improves the hydrophilic capacity of PAA through the increase of the polymeric PAA chain length. Moreover, it decreases the dissolution behaviour of the free applicable PAA into soils, where PAA is highly soluble in water.

By using a $0.2 \mathrm{wt} \%$ of soil stabilizer, PAA-soil aggregates were collapsed after 15 min, while PAA-LDH soil aggregates were collapsed after $96 \mathrm{~min}$. With the increase of soil stabilizer-concentration to $0.4 \mathrm{wt} \%$ and $0.6 \mathrm{wt} \%$, the collapsing time of the aggregates was increased. The PAA-treated soil-aggregates showed an explosive collapse to the initial integrity of soil, while the PAA-LDH modified-aggregates showed a gradual collapse. The collapse of PAA-LDH soil-aggregates is characterized by an initial collapse on the edges of the aggregates followed by a slow collapse rate into secondary aggregates of smaller size, and finally the secondary aggregates were collapsed. In conclusion, PAA-LDH modification stabilized the soil aggregates and improved the water-stability.

\section{References}

1. K. M. Raju, M. P. Raju and Y. M. Mohan, J. Appl. Polym. Sci. 85, 1795 (2001).

2. P. Chen, W. Zhang, W. Luo and Y. Fang, J. Appl. Polym. Sci. 93, 1748 (2004).

3. S. Ma, M. Liu and Z. Chen. J. Appl. Polym. Sci. 93, 2532 (2004).

4. J. W. Boclair, P. S. Braterman, B. D. Brister and F. Yarberry, Chem. Mater. 11 (8), 2199 (1999).

5. L. P. Cardoso, R. Celis, J. Cornejo and J. B. Valim, J. Agric. Food Chem. 54, 5968 (2006).

6. I. H. Hafez, M. R. Berber, K. Minagawa, T. Mori and M. Tanaka. J. Agric. Food Chem. 58, 10118 (2010).

7. S. Miyata, Clays Clay Miner. 23 (1), 369 (1975).

8. Y. Lin, J. Wang, D. G. Evans and D. Li, J. Phys. Chem. Solids 67 (5-6), 998 (2006).

9. J. Olanrewaju, B. L. Newalkar, C. Mancino, and S. Komarneni, Mater. Lett. 45 (6), 307 (2000).

10. S. Dubinsky, G. S. Grader, G E. Shter, M S. Silverstein, Polym. Degrad. Stab. 86, 171 (2004). 